Modeling, Identification and Control, Vol. 40, No. 1, 2019, pp. 27-39, ISSN 1890-1328

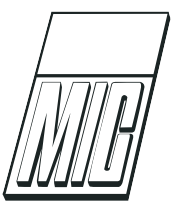

\title{
Control System Commissioning of Fully Levitated Bearingless Machine
}

\author{
P. Jaatinen ${ }^{1}$ J. Vuojolainen ${ }^{1}$ N. Nevaranta ${ }^{1}$ R. Jastrzebski ${ }^{1}$ O. Pyrhönen ${ }^{1}$ \\ ${ }^{1}$ Department of Electrical Engineering, Lappeenranta University of Technology, FI-53851 Lappeenranta, Finland \\ E-mail: pekko.jaatinen@lut.fi, jouni.vuojolainen@lut.fi, niko.nevaranta@lut.fi, rafal.jastrzebski@lut.fi, \\ olli.pyrhonen@lut.fi
}

\begin{abstract}
The bearingless permanent magnet synchronous motor (BPMSM) is a compact motor structure that combines the motoring and bearing functions based on well-designed integrated windings for generating both torque and magnetic suspension force. In order to achieve a successful high-performance control design for the BPMSM, an adequate model of the rotor dynamics is essential. This paper proposes simplified multiple-input and multiple-output (MIMO) control approaches, namely the pole placement and the linear-quadratic regulator (LQR), that allow to carry out identification experiments in full levitation. Additionally, the stability of the MIMO levitation controller is verified with the rotation tests. Compared with other recently published works, the novelty of this paper is to experimentally demonstrate that a stable fully levitated five-degrees-of-freedom (5-DOF) operation of a bearingless machine can be achieved by the proposed approach, and thereby, options for commissioning of such a system are obtained.
\end{abstract}

Keywords: Bearingless, magnetic levitation, MIMO control, self-levitating, system identification, 5-DOF

\section{Introduction}

Operation in the high-speed region is very beneficial especially in the field of compressor applications. The compressor pressure ratio and mass flow rate can be raised by increasing the rotational speed Yoon et al. (2013). In the speed range of $20000 \mathrm{r} / \mathrm{min}$ and over, the electrical motor efficiency can be increased by achieving the minimum weight-power ratio. It is clear that operating in the high-speed region increases both the motor and compressor efficiency. Nowadays there is a growing interest in high-speed technology, where the traditional bearing solution is replaced by a more advanced solution, namely active magnetic bearings (AMBs) Gerhard Schweitzer (2009). The wellknown benefits of AMBs are contact-free operation, active control of the rotor, and self diagnostic properties. As AMBs do not need oil lubrication because of the magnetic levitation of the rotor, they are the most suitable solution for oil-free compressor applications in the fields of pharmacy and food industry. However, one drawback of the AMBs is that they extend the total length of the rotor as the radial and axial magnetic bearings need a certain amount of space, which results in an increased axial length of the rotor shaft along with a larger and more complicated motor structure. Depending on the operational speed and rotor mechanical dimensions, this extra length can lower the flexible mode frequencies to the operating region. This is an unwanted feature as the operation close to the flexible mode is difficult. From the viewpoint of the overall system behavior, and especially with respect to controllability, it is advantageous that the rotor does not need to pass flexible modes.

Reducing the rotor length, simultaneously keeping the benefits of the traditional AMBs, a self-levitating or bearingless motor technology can be applied Chiba et al. (2009). In a bearingless motor, one stator pro- 
duces both the levitation force to support the rotor and the torque for rotation. This can be achieved by the use of separate windings or by different common winding configurations in one stator unit Chiba et al. (2013). Because the windings are of a three-phase type for both generating torque and levitation force, commercial motor drives can be used, and thus, the amount of power electronics is decreased compared with the traditional AMB configuration.

Bearingless operation is possible also with singlestator disc-shape motors Mitterhofer and Amrhein (2012). Other applications that exploit the benefits of bearingless operation are artificial hearts Hoshi et al. (2006) and canned pump Warberger et al. (2010) applications, where a long air gap length is needed. However, in this paper, a standard horizontal-type machine equipped with two bearings is considered. The machine type with two or more supporting bearings can handle a higher loading force caused by the weight and mass flow of the impeller wheel.

In general, when dealing with high-speed machines, it is important to analyze the rotor behavior Swanson et al. (2008). As a result of the dynamic properties of the rotor structure, bending occurs when the rotation speed is increased. Without qualitative analysis of the rotor dynamics, the rotor operating point in nominal operation can be close to the rotor flexible mode. Thus, it is of great importance that in the machine commissioning phase, the rotor dynamics are identified in order to verify the flexible modes of the rotor Noh et al. (2017). A common method is to use an impulse hammer with vibration sensors to conduct the mode analysis. Naturally, as the AMB system is equipped with a displacement sensor and power electronics, the rotor identification can be made in the system without removing the rotor.

In recent years, a variety of different bearingless machine setups have been introduced in the literature, and their control has become a topic of significant interest. The five-degrees-of-freedom (5-DOF) control of a bearingless machine has been reported in Takemoto et al. (2009); Yamamoto et al. (2011); Severson et al. (2017), and other studies have considered the combination of a bearingless motor and a magnetic bearing in Cao et al. (2017); Schneider and Binder (2007). Here, the 5-DOF operation refers to two radial xy-planes and one axial z-plane of the control axes. Note, however, that many of the reported prototypes are laboratory versions, where all degrees of freedom (DOF) have not been evaluated. It is also worth emphasizing that in these examples the most common structure is a bearingless motor with a ball bearing supporting the other end of the rotor Chiba et al. (2013); Sun et al. (2016a); Ooshima et al. (2015); Yang et al. (2010); Huang et al.
(2014). Although there are a few publications where the system has one bearingless motor, it is not shown or reported how the conical movement of the rotor is stabilized Qiu et al. (2015); Sun et al. (2016b); Xue et al. (2015); Yang and Chen (2009); Chen and Hofmann (2011); Cao et al. (2016); Zhang et al. (2016); Zhao and Zhu (2017). In addition, a common factor in all these publications is that they apply PID-based position controllers. To the authors' knowledge, only the model-based controller has been addressed in Messager and Binder (2016) for machines of the horizontal dual bearingless motor type. Another approach based on a linear-quadratic regulator (LQR) controller for a bearingless motor has been introduced in Kauss et al. (2008). However, the presented prototype is 2-DOF and the other end is supported by a ball bearing.

In order to conduct rotor identification, the rotor must be fully levitated. The aim of this paper is to study MIMO control approaches that provide a stable fully levitated operation of a bearingless machine. The novelty of this paper compared with the previously reported studies is that it provides experimental results that show the actual 5-DOF operation of a bearingless machine, and more importantly, introduces results of the full levitation. For this purpose, a 4-DOF MIMO controller is used for the radial position control. The axial position is controlled with an axial AMB, and it is separated from the radial controller. Rigid body is used as an initial rotor model. Pole placement and LQR radial position controllers are used, and the suitability of the controllers is discussed. The designed 4-DOF radial controllers are simulated and tested in a $10 \mathrm{~kW}$ dual motor interior permanent magnet bearingless machine. Additionally, the stability of the levitation control is verified with low-speed rotation tests. Finally, system identification experiments are carried out with the pole placement and the LQR controller by superposing a stepped sine excitation signal to the system.

\section{Problem statement}

To operate in a high-speed region, the dynamic properties of the rotor must be known. An initial analysis of the rotor dynamics is normally done with analytical tools, by which the natural frequencies of the rotor are found. However, experimental tests are mandatory to verify the model and detect possible defects of the rotor. One common method to carry out experimental modal analysis is to use an impulse hammer, which includes for example an integral piezoelectric accelerometer sensor to produce the excitation to the rotor and measure the applied force Kolondzovski et al. (2010). When the rotor system is equipped with AMBs, the 
same modal analysis can be done in the system. Similarly as in the impulse hammer test, the AMBs produce the excitation signal and displacement sensors are used to measure the vibration of the rotor. Based on the results, the natural frequencies of the rotor can be found. The obtained results can be used to improve the analytical model by updating the rigid and flexible modes, thereby resulting in a more accurate system model.

To simplify this procedure in a bearingless machine, the rotor can be levitated without a rotating field as the rotor identification is made at a standstill. In this case, the decoupling of the torque and levitation windings can be ignored. When knowing the rotor angle and transforming the three-phase windings into a 2-phase system, the control principles of traditional AMB systems can be adopted.

\subsection{System description}

The prototype machine consists of two identical interior permanent magnet (IPM) bearingless motors (BMs) together with an axial magnetic bearing. Fig. 1. depicts the prototype machine. The axial magnetic bearing is in the middle of the machine, and bearingless motors are placed on opposite sides of the machine. This provides a symmetrical rotor structure when the load is not considered. A block diagram of the full control system is shown in Fig. 2. The rotor position is measured with an eddy-current sensor differentially from the radial direction and single ended from the axial direction. A non-contact encoder is placed on the right side of the machine to sense the rotor angle. Moreover, five industrial motor drives are used to operate the machine: one is needed for the axial bearing and two for the torque and radial force production for each BM. Each motor drive includes a field programmable gate array (FPGA), where the inner loop current controller is implemented. A block diagram of the inner current control loop is illustrated in Fig. 3. The upperlevel control is implemented in a Beckhoff industrial $\mathrm{PC}$, and the communication between the industrial PC and the motor passes through an EtherCAT industrial fieldbus. The sampling time of the control system is $50 \mu \mathrm{s}$.

\section{Model of the system}

In this paper, the rigid rotor model is used to tune the proposed control approaches. In general, a mathematical model of the system can be presented using a state-space representation

$$
\begin{aligned}
& \dot{\mathbf{x}}(t)=\mathbf{A} \mathbf{x}(t)+\mathbf{B u}(t) \\
& \mathbf{y}(t)=\mathbf{C x}(t)
\end{aligned}
$$

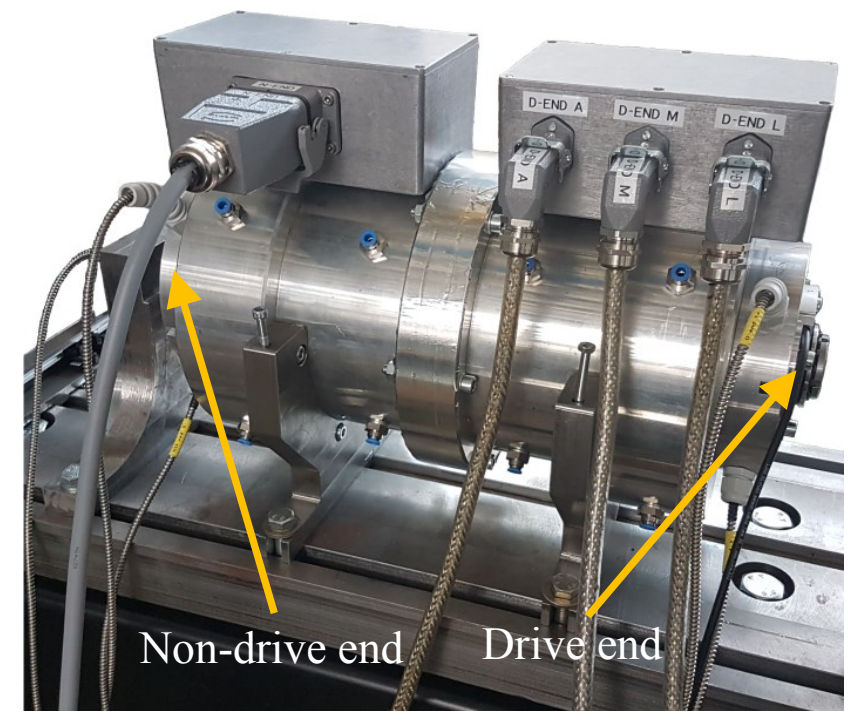

Figure 1: Photograph of the $10 \mathrm{~kW}$ dual motor bearingless machine. The axial AMB is in the middle of the machine and bearingless motors are located on both ends.

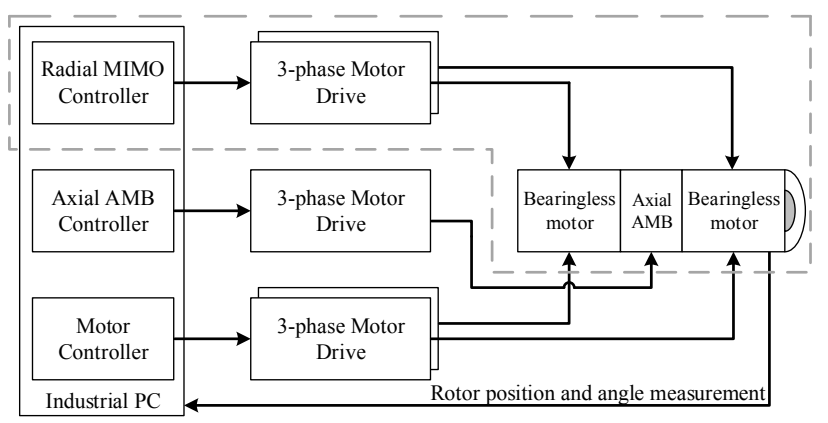

Figure 2: Block diagram describes the overview of the system configuration. All controllers are implemented on a Beckhoff industrial PC shown in far left. In total, five 3-phase motor drives are used to produce levitation force and torque. Three drives are allocated for the 5-DOF levitation purposes, and both motors are driven separately. The rotor position in 5 -DOF is measured together with the rotor angular position.

where $\mathbf{A}$ is the system matrix, $\mathbf{B}$ is the input matrix, and $\mathbf{C}$ is the output matrix. The vectors $\mathbf{x}$ and $\mathbf{u}$ are state and input vectors, respectively. In this paper, separate models for the axial and radial directions are used as the coupling is not strong. In the axial direction, the rotor is modeled as a point mass, whereas in the radial direction, a rigid body rotor model is used. 


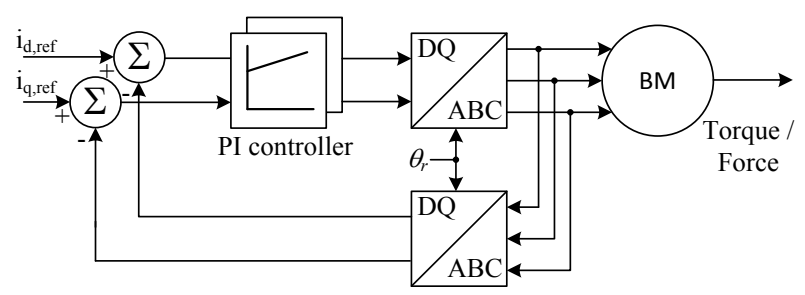

Figure 3: Block diagram of the PI current controller scheme applied to motor drives that produce the radial force. $\mathrm{ABC}$ : three-phase reference frame and DQ: rotor reference frame.

\subsection{Rotor model}

General form of the rotor model is presented in the following:

$$
\mathbf{M} \ddot{\mathbf{q}}(t)+(\mathbf{D}+\Omega \mathbf{G}) \dot{\mathbf{q}}(t)+\mathbf{K q}(t)=\mathbf{F}(t),
$$

where $\mathbf{M}$ is the mass matrix, $\mathbf{D}$ is the damping matrix, $\Omega$ is the rotational speed, $\mathbf{G}$ is the gyroscopic matrix, $\mathbf{K}$ is the stiffness matrix, $\mathbf{F}$ is the force applied to the rotor and $\mathbf{q}$ is the displacement vector of the rotor. This model can be simplified to a rigid rotor model, which describes the rotor movement with respect to the center of the rotor mass Smirnov (2012)

$$
\mathbf{M} \ddot{\mathbf{q}}(t)+\Omega \mathbf{G} \dot{\mathbf{q}}(t)=\mathbf{F}(t),
$$

where $\mathbf{M}$ is the diagonal matrix including rotor mass and inertia at the center of mass, $\mathbf{q}=$ $\left[\begin{array}{llll}x & y & \alpha_{x} & \alpha_{y}\end{array}\right]^{T}$ is the vector that describes the rotor position in the $x y$-axis and the angle around the corresponding axis at the center of mass. As the displacement sensors and the magnetic bearings are not located at the center of mass, a coordinate transformation is needed for the control design and simulation purposes. To acquire the absolute location in the $x y$-axis of the sensors, $\mathbf{q}_{s}=$ $\left[\begin{array}{llll}x_{D, s} & y_{D, s} & x_{N D, s} & y_{N D, s}\end{array}\right]^{T}$ and the magnetic bearing locations, $\mathbf{q}_{b}=\left[\begin{array}{llll}x_{D, b} & y_{D, b} & x_{N D, b} & y_{N D, b}\end{array}\right]^{T}$ at the drive and non-drive end of the machine, the following transformation matrices are applied

$\mathbf{q}_{b}=\underbrace{\left(\begin{array}{cccc}1 & 0 & 0 & -a \\ 0 & 1 & -a & 0 \\ 1 & 0 & 0 & b \\ 0 & 1 & b & 0\end{array}\right)}_{\mathbf{T}_{b}} \mathbf{q}, \quad \mathbf{q}_{s}=\underbrace{\left(\begin{array}{cccc}1 & 0 & 0 & -c \\ 0 & 1 & -c & 0 \\ 1 & 0 & 0 & d \\ 0 & 1 & d & 0\end{array}\right)}_{\mathbf{T}_{s}} \mathbf{q}$,

where $a, b$ are the drive-end and non-drive-end bearing locations from the center of the rotor mass, respectively and $c, d$ are the drive-end and non-drive-end sensor locations from the center of mass. Rotor cross-sectional view is illustrated in Fig. 4.

Radial forces produced by the bearingless machine can be presented by the following equation

$$
\mathbf{F}(t)=\mathbf{K}_{x} \mathbf{q}_{b}+\mathbf{K}_{i} \mathbf{i}_{c},
$$

where $\mathbf{F}$ is the total linearized radial force generated by the bearingless machine, $\mathbf{q}_{b}$ is the rotor position at the bearing location, $\mathbf{i}_{c}$ is the control current to the levitation windings, $\mathbf{K}_{x}$ is the diagonal position stiffness matrix, and $\mathbf{K}_{i}$ is the diagonal current stiffness matrix. The total force depends on the rotor position and current in the levitation windings. The coefficients $\mathbf{K}_{x}$ and $\mathbf{K}_{i}$ can be determined experimentally by different tests and measurements. Parameters of the prototype machine are listed in Table 1.

In Fig. 5 a) the position stiffness value is determined by moving the rotor in the air gap, and the force caused by the unbalance pull of the permanent magnets is measured. From this measurement, the slope of the position stiffness can be calculated, $\mathbf{K}_{x}=\triangle f_{x} / \triangle P_{x}$. The current stiffness is measured by applying current in the levitation windings and measuring the corresponding radial force. Similarly, from the measured slope, the current stiffness can be calculated, $\mathbf{K}_{i}=\triangle f_{x} / \triangle i_{L}$. It can be seen that the measured values are closely matching the FEM simulations presented in Fig. 5. Measured values are used in the control design. The force measurement setup is described in more detail in Jaatinen et al. (2016).

The rigid rotor model presented in (3) can be further simplified by neglecting the gyroscopic matrix as the rotor is not rotating during the identification, that is, $\Omega=0$. Furthermore, this simplification is also valid for the rotating system when axial length of the rotor is much greater than the rotor diameter thus the gyroscopic effect is then negligible Gerhard Schweitzer (2009). By substituting (4) and (5) into (3), a simplified rigid rotor model is achieved

$$
\mathbf{M}_{b} \ddot{\mathbf{q}}_{\mathbf{b}}=\mathbf{K}_{x} \mathbf{q}_{b}+\mathbf{K}_{i} \mathbf{i}_{c}
$$

where $\mathbf{M}_{b}=\left(\mathbf{T}_{b}^{-1}\right)^{T} \mathbf{M} \mathbf{T}_{b}^{-1}$ is the mass matrix in the bearing plane. In the state-space form, the simplified rotor model is written as

$$
\begin{aligned}
\mathbf{A}_{r} & =\left[\begin{array}{cc}
\mathbf{0} & \mathbf{I} \\
\left(\mathbf{M}_{b}\right)^{-1} \mathbf{K}_{x} & \mathbf{0}
\end{array}\right], \\
\mathbf{B}_{r} & =\left[\begin{array}{c}
\mathbf{0} \\
\left(\mathbf{M}_{b}\right)^{-1} \mathbf{K}_{i}
\end{array}\right], \\
\mathbf{C}_{r} & =\left[\begin{array}{ll}
\mathbf{T}_{\mathbf{s}} \mathbf{T}_{\mathbf{b}}^{-\mathbf{1}} & \mathbf{0}
\end{array}\right] .
\end{aligned}
$$




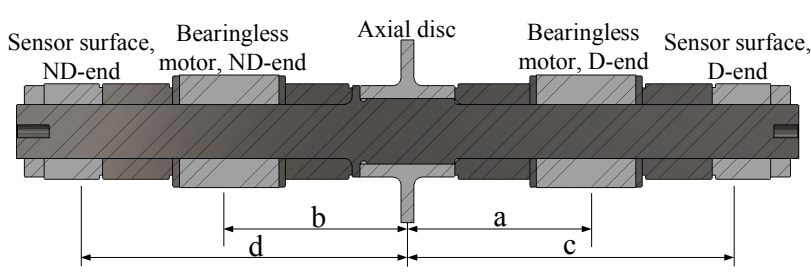

Figure 4: Cross-sectional view of the rotor in the prototype system. Locations of the bearingless motors and the sensor surfaces are measured respect of the center of mass.
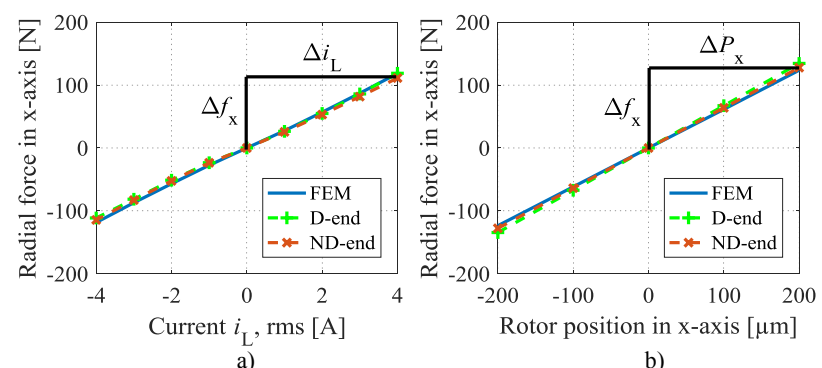

b)

Figure 5: Simulated and measured current and position stiffnesses. The current stiffness can be calculated from the slope presented in a). In the same manner, the position stiffness can be calculated from the slope presented in b).

\subsection{Actuator model}

The actuator consists of the dynamics of the inner current control loop. A straightforward method to model the actuator dynamics is to use the bandwidth of the current controller

$$
G_{a}=\frac{\omega_{b w}}{s+\omega_{b w}}
$$

where $\mathbf{G}_{a}$ is the approximate transfer function of the inner current loop and $\omega_{b w}$ is the bandwidth of the current controller.

In the simulation, the inner control loop consists of the PI controller, the motor drive model, and the bearingless motor model including the levitation windings. The motor drive is modeled as two-stage switching with a pulse width modulator. The bearingless motor is modeled in the $d q$ reference frame as

$$
\begin{aligned}
& u_{d}=R i_{d}+\frac{d}{d t} L_{d} i_{d}-\omega L_{q} i_{q}, \\
& u_{q}=R i_{q}+\frac{d}{d t} L_{q} i_{q}+\omega L_{d} i_{d}
\end{aligned}
$$

where $u$ is the voltage over the levitation windings, $R$ is the resistance of the levitation windings, $L$ is the inductance of the levitation windings, $i$ is the current of the levitation windings, and $\omega$ is the electrical angle.

\subsection{Full model}

A full model can be produced by combining the rotor model with the actuator model.

$$
\begin{aligned}
& \mathbf{A}=\left[\begin{array}{cc}
\mathbf{A}_{a} & \mathbf{0} \\
\mathbf{B}_{r} \mathbf{C}_{a} & \mathbf{A}_{r}
\end{array}\right], \quad \mathbf{B}=\left[\begin{array}{c}
\mathbf{B}_{a} \\
\mathbf{0}
\end{array}\right], \\
& \mathbf{C}=\left[\begin{array}{ll}
\mathbf{0} & \mathbf{C}_{r}
\end{array}\right],
\end{aligned}
$$

where $\mathbf{B}_{a}=-\mathbf{A}_{a}=\operatorname{diag}\left[\begin{array}{llll}\omega_{b w} & \omega_{b w} & \omega_{b w} & \omega_{b w}\end{array}\right]$ is the current controller bandwidth, and the rigid rotor model matrices are denoted by the subscript $r$.

\subsection{Axial AMB model}

The axial direction of the rotor can be controlled separately as the coupling to the radial direction is negligible in the center of the air gap. As the axial AMB controls only 1-DOF, the model of the rotor can be simplified to a point mass model

$$
m \ddot{q}=K_{x} q_{a}+K_{i} i_{c}
$$

where $m$ is the rotor mass, $q_{a}$ is the acceleration of the rotor, $K_{i}$ is the current stiffness, and $K_{x}$ is the position stiffness.

\section{MIMO control of a bearingless machine}

In the literature, there are many publications that address the issues of the MIMO control of traditional AMB systems equipped with two radial and one axial AMBs Yoon et al. (2013); Gerhard Schweitzer (2009). The same principles can be adopted to the bearingless machine control. However, there are two major differences compared with the traditional AMB system. Firstly, the rotating magnetic flux that generates the levitating force is synchronous with the rotor rotation. Secondly, decoupling of the motor control from the levitation control is required. If the decoupling parameters are correctly identified, the motor control does not affect the performance of the levitation controller Ooshima et al. (2004). It is emphasized that in this paper, the decoupling controller is not taken into consideration as the rotor identification is conducted with a nonrotating rotor. Moreover, a 4-DOF MIMO radial controller with a PID-type axial controller for commissioning and rotor identification purposes is tuned based on a rigid rotor model. 
Table 1: Machine parameters

\begin{tabular}{llll}
\hline \hline Parameter & Symbol & Value & Unit \\
\hline Nominal speed & $\Omega_{\mathrm{nom}}$ & 30000 & $\mathrm{r} / \mathrm{min}$ \\
Nominal power per motor unit & $P_{\mathrm{nom}}$ & 5 & $\mathrm{~kW}$ \\
Rotor mass & $m$ & 11.65 & $\mathrm{~kg}$ \\
Rotor inertia & $J$ & 0.232 & $\mathrm{kgm}$ \\
Resistance, levitation winding & $R$ & 0.27 & $\Omega$ \\
Inductance, levitation winding & $L$ & 3.27 & $\mathrm{mH}$ \\
BM location & $a, b$ & 107.5 & $\mathrm{~mm}$ \\
Position sensor location & $c, d$ & 211 & $\mathrm{~mm}$ \\
Air gap length & $l_{\delta}$ & 0.6 & $\mathrm{~mm}$ \\
Rotor length & $l_{\mathrm{r}}$ & 480 & $\mathrm{~mm}$ \\
BM lamination stack length & $l_{\mathrm{rl}}$ & 61 & $\mathrm{~mm}$ \\
BM lamination diameter & $d_{\mathrm{rl}}$ & 68.8 & $\mathrm{~mm}$ \\
BM stator outer diameter & $d_{\mathrm{s}}$ & 150 & $\mathrm{~mm}$ \\
Axial disk thickness & $l_{\mathrm{a}}$ & 8 & $\mathrm{~mm}$ \\
Axial disk diameter & $d_{\mathrm{a}}$ & 112 & $\mathrm{~mm}$ \\
Rotor shaft diameter & $d_{\mathrm{rs}}$ & 33 & $\mathrm{~mm}$ \\
Current stiffness, measured & $K_{\mathrm{i}}$ & 29 & $\mathrm{~N} / \mathrm{A}$ \\
Position stiffness, measured & $K_{\mathrm{x}}$ & 672 & $\mathrm{~N} / \mathrm{mm}$ \\
Current stiffness, FEM & $K_{\mathrm{i}, \mathrm{FEM}}$ & 29.6 & $\mathrm{~N} / \mathrm{A}$ \\
Position stiffness, FEM & $K_{\mathrm{x}, \mathrm{FEM}}$ & 618 & $\mathrm{~N} / \mathrm{mm}$ \\
Maximum input deviation & $u_{n m a x}$ & 2 & $\mathrm{~A}$ \\
Maximum output deviation & $m_{\mathrm{n}}$ & 25 & $\mu \mathrm{m}$ \\
\hline \hline & & & \\
\hline
\end{tabular}

Furthermore, an additional coordinate transformation is needed when comparing the bearingless system with the traditional AMB system. In Fig. 6, the principle of the radial force generation both in the $x$ and $y$ directions is shown. Here, the three-phase winding is transformed into a two-phase presentation. When the rotor is in a certain angular position, for instance $0 \mathrm{deg}$, where the poles are parallel with the stationary $x y$-reference frame, the corresponding two-phase current produces force in that axis. By changing the polarity of the current, the force direction can be reversed. By taking into account the rotation of the rotor in the coordinate transformation, the force can be generated at any angle. A radial position control-loop block diagram is presented in Fig. 7. Note the coordinate transformation between the position controller and the inner current controller.

\subsection{State-feedback control with pole placement}

One common control method for handling state equations is state feedback with pole placement, in which the locations of the closed-loop poles are selected to ob- tain the desired performance. As all states are not measurable, a state estimator is also needed. To remove the steady-state error, an integral state is augmented to the state feedback controller. The full discrete-time state equation can be written Franklin et al. (2010)

$\left[\begin{array}{c}\mathbf{x}(k+1) \\ \mathbf{x}_{I}(k+1)\end{array}\right]=\left[\begin{array}{cc}\mathbf{\Phi} & \mathbf{0} \\ \mathbf{C} & \mathbf{I}\end{array}\right]\left[\begin{array}{c}\mathbf{x}(k) \\ \mathbf{x}_{I}(k)\end{array}\right]+\left[\begin{array}{c}\boldsymbol{\Gamma} \\ \mathbf{0}\end{array}\right] \mathbf{u}(k)-\left[\begin{array}{l}\mathbf{0} \\ \mathbf{I}\end{array}\right] \mathbf{r}(k)$,

where $\boldsymbol{\Phi}, \boldsymbol{\Gamma}$ are discretized system state and input matrices, $\mathbf{C}$ is the output matrix, $\mathbf{I}$ is the identity matrix, $\mathbf{x}$ is the system state vector, $\mathbf{x}_{I}$ is the integral state vector, $\mathbf{u}$ is the system input vector, and $\mathbf{r}$ is the reference input vector. The feedback law is then written as

$$
\mathbf{u}(k)=-\left[\begin{array}{ll}
\mathbf{K} & \mathbf{K}_{\mathbf{I}}
\end{array}\right]\left[\begin{array}{c}
\mathbf{x}(k) \\
\mathbf{x}_{I}(k)
\end{array}\right]
$$

where $\mathbf{K}$ is the state feedback gain and $\mathbf{K}_{I}$ is the integrator gain.

The state estimator uses the following presentation

$$
\hat{\mathbf{x}}(k+1)=\boldsymbol{\Phi} \hat{\mathbf{x}}(k)+\boldsymbol{\Gamma} \mathbf{u}(k)+\mathbf{L}(\mathbf{y}(k)-\mathbf{C} \hat{\mathbf{x}}(k)),
$$

where $\hat{\mathbf{x}}$ is the estimated state vector and $\mathbf{L}$ is the feedback gain of the state estimator. In this paper, the state feedback controller is designed by using the principles presented in Gerhard Schweitzer (2009).

The main drawback of the pole-placement-based tuning is that it is not very intuitive. Secondly, when the system degree increases, also the number of poles to be placed increases, resulting in a more complex tuning problem. This is an important factor to be acknowledged, especially when including flexible modes to the control model.

\subsection{Linear-quadratic regulator}

There are other control methods that facilitate controller tuning by providing more intuitive tools, which do not need direct manipulation of the poles. One of these optimal control methods is the linear-quadratic regulator (LQR). The controller tuning is based on minimization of the quadratic cost function

$$
\mathcal{J}=\frac{1}{2} \sum_{k=0}^{N}\left[\mathbf{x}^{T}(k) \mathbf{Q}_{1} \mathbf{x}(k)+\mathbf{u}^{T}(k) \mathbf{Q}_{2} \mathbf{u}(k)\right]
$$

where $\mathcal{J}$ is the cost function, $\mathbf{x}$ is the state vector, $\mathbf{u}$ is the input vector, $\mathbf{Q}_{1}$ is the output weight function, and $\mathbf{Q}_{2}$ is the input weight function. The weighting 


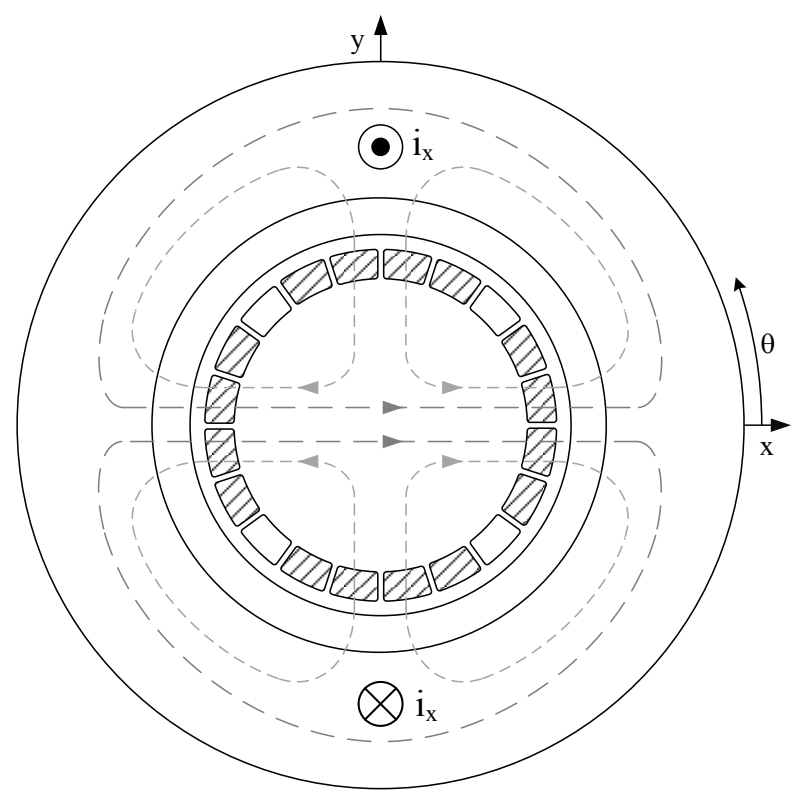

a)

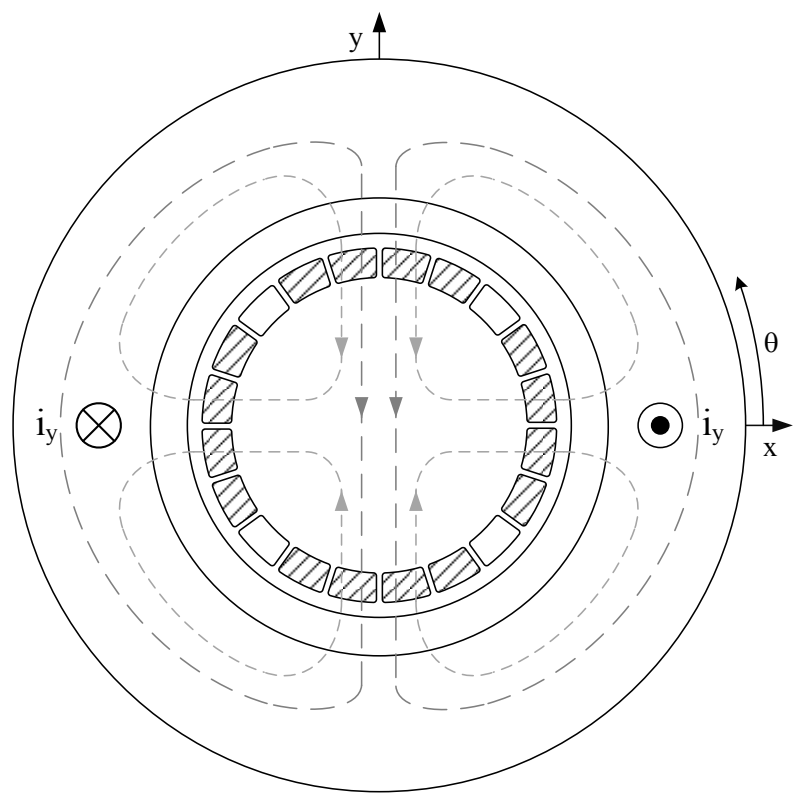

b)

Figure 6: Description of radial force generation in a permanent magnet bearingless motor. The three-phase windings are transformed into a two-phase presentation in the $x y$ plane. The currents of the two-phase windings are denoted by $i_{x}$ and $i_{y}$. The principle of producing radial force in the $x$-axis is shown in a). By applying current to the $\mathrm{x}$ phase windings, the flux is increased and decreased opposite to the air gap in $x$-axis. This flux unbalance produces the radial force. By applying negative current, the force direction can be reversed. In a similar fashion, the radial force in the $y$-axis can be produced by applying current in the $y$-phase winding.

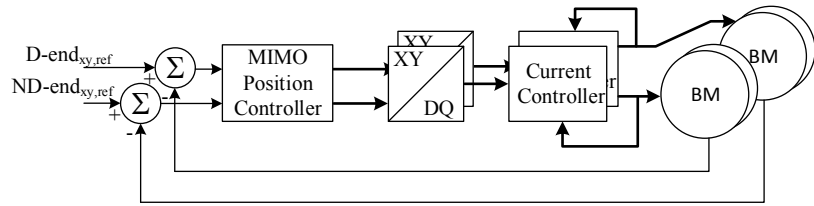

Figure 7: Block diagram of the position control loop.

functions are diagonal matrices that affect the states and inputs of the system. There are different methods to determine the weighting functions $\mathbf{Q}_{1}$ and $\mathbf{Q}_{2}$. One of the methods is called Bryson's rule Franklin et al. (2010), where the effect of the state weight on the output follows

$$
\mathbf{Q}_{1}=\mathbf{C}^{T} \overline{\mathbf{Q}}_{1} \mathbf{C}
$$

The weights are selected for the output by deciding how large a deviation of the output is acceptable

$$
\overline{\mathbf{Q}}_{1, n}=\left(\begin{array}{cccc}
1 / m_{1}^{2} & 0 & \cdots & 0 \\
0 & 1 / m_{2}^{2} & \cdots & 0 \\
\vdots & \vdots & \ddots & \vdots \\
0 & 0 & \cdots & 1 / m_{n}^{2}
\end{array}\right)
$$

where $m_{n}$ is the maximum deviation of the output signal. The weights for the inputs are selected by the maximum input signal amplitude

$$
\mathbf{Q}_{2, n}=\left(\begin{array}{cccc}
1 / u_{1 \max }^{2} & 0 & \cdots & 0 \\
0 & 1 / u_{2 \max }^{2} & \cdots & 0 \\
\vdots & \vdots & \ddots & \vdots \\
0 & 0 & \cdots & 1 / u_{\text {max }}^{2}
\end{array}\right)
$$

where $u_{n \max }$ is the maximum input signal deviation.

Table 1 lists the values selected for the weights $m$ and $u$ based on several simulation iterations.

When designing the LQR-based controller, the degree of freedom is lower (two parameters) than with the pole placement method, where eight poles have to 


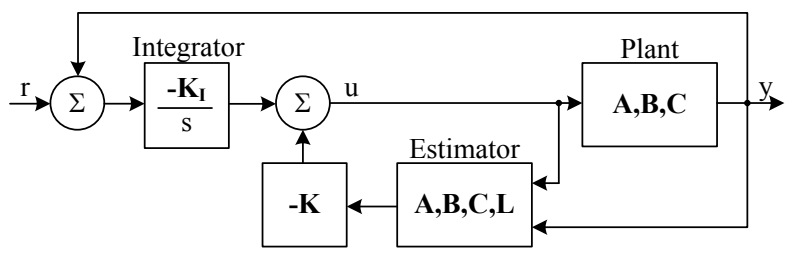

Figure 8: Block diagram of the state feedback controller. A state estimator is necessary for generating the full state vector including rotor acceleration, which is not measurable. Naturally, an integral action is added to remove the steady-state error.

be selected. This difference is amplified in the case of the flexible plant model, where more states are added to the system plant.

\section{Experimental Results}

Both controllers are tested with the prototype bearingless machine. First, the initial lift-up test is conducted and compared with simulations in Fig. 9. The rotor position is shown during the initial lift-up with the pole placement and the LQR controller. At the beginning of the test, the rotor is resting on the backup bearings, where it is levitated to the center of the air gap. Based on the simulations, it can be noted that the pole placement controller has a higher overshoot, but both controllers provide full levitation.

In Figs. 10 and 11, the current in the dq reference frame is shown for the pole placement controller and the LQR controller during the rotor lift-up sequence. Because of the unbalanced magnetic pull of the embedded magnets in the rotor, a high current peak is needed to lift the rotor away from the backup bearings to the center of the air gap. Based on the results, it can be concluded that both of the proposed controllers meet the requirement of levitating the rotor. It can also be seen that a good correspondence between the simulations and measurements is achieved. From the current RMS values in the steady-state situation we can notice that the LQR controller provides lower current demand. It is pointed out, however, that there is one notable difference between the BMs in the experimental test; the ND-end has a smaller current ripple than the D-end.

\subsection{System Identification}

As was shown in Fig. 9, both the proposed control approaches provided a stable fully levitated operation of the bearingless machine. Thus, system identification
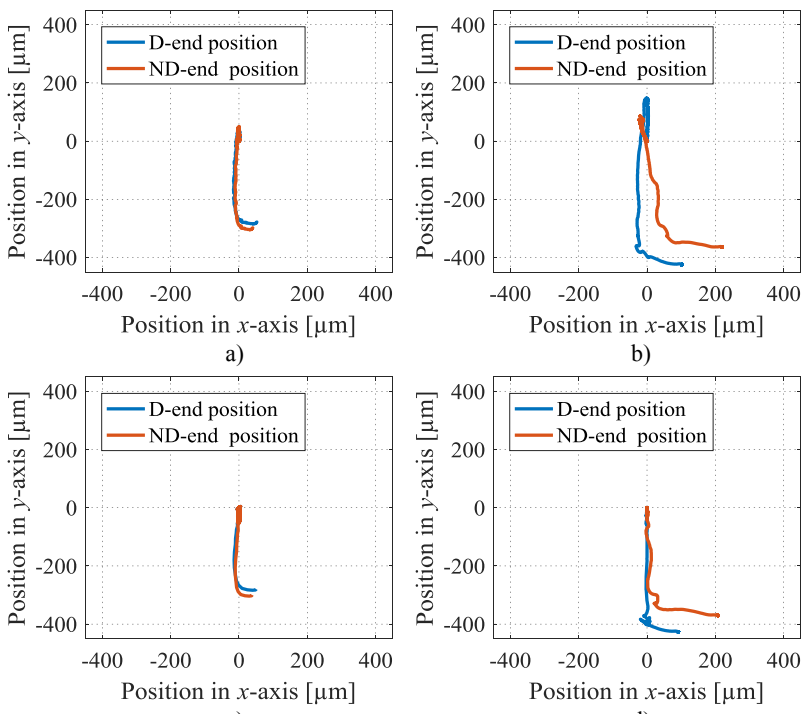

c)

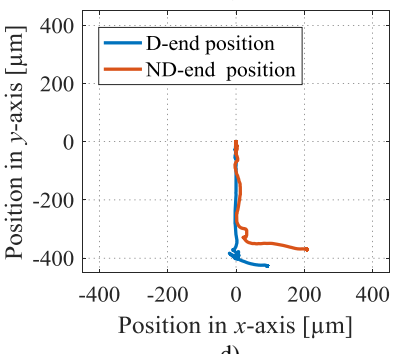

d)

Figure 9: Simulated and measured rotor lift-up from the backup bearings. Initially, the rotor is lying on the backup bearings, and after the controller is enabled, the rotor is magnetically levitated to the operating point, that is the origin $(x, y)=(0,0)$. Simulation and measurement results for the pole placement controller are shown in a) and b), and for the LQR controller in c) and d).

experiments can be carried out when the rotor is levitating by superposing artificially generated excitation signals to the control system. In this paper, an adaptive amplitude stepped sine signal is considered with a frequency band from $1 \mathrm{~Hz}$ to $750 \mathrm{~Hz}$ in order to validate the suitability of the control approaches for commissioning purposes. System identification experiments are carried out with both control approaches. In Fig. 12, the experimentally obtained frequency responses are shown. Uncertainty is shown in the low frequency area $(<10 \mathrm{~Hz})$ as it is challenging to identify the DC-area with the motor inverter. Also the closed loop controller influences to the low frequency region limiting the accuracy of identification. Nevertheless, identified rotor model for both controllers is matching closely to the initial rigid rotor model. Identified rotor model can be further use in the control design where the flexible part is included. Evidently, the system rotor dynamics can be identified in the full-levitation mode similarly as with the 5-DOF AMB system Vuojolainen et al. (2017). 


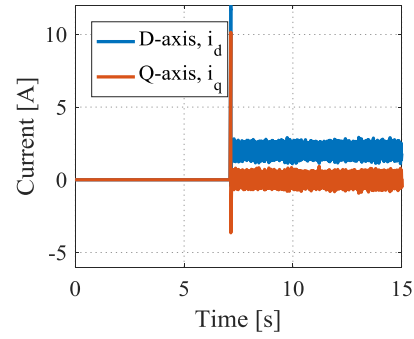

a)

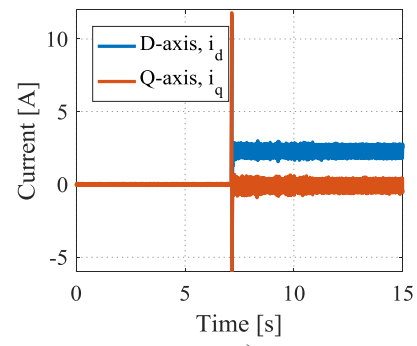

c)

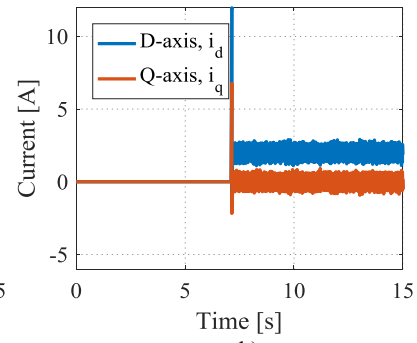

b)

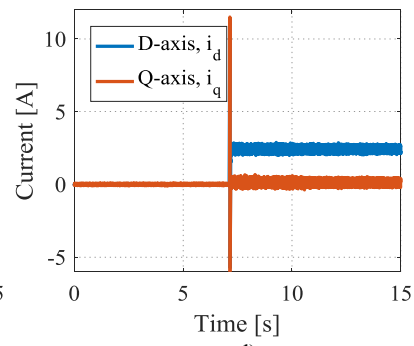

d)

Figure 10: Simulated and measured DQ currents during the rotor lift-up with the pole placement controller. Simulation of the DQ axis current of the D-end and the ND-end motor are shown in a) and b), respectively. The measured DQ axis current of the D-end and the ND-end motor are shown in c) and d), respectively. Steady state RMS values for current in the simulation a) and b) are $2 \mathrm{~A}$. For the measured steady state RMS current values c) $2.3 \mathrm{~A}$ and d) $2.4 \mathrm{~A}$.

\subsection{Rotational tests}

To further validate the observations reported in this paper, rotational tests are carried out with modest velocity of 150 and $300 \mathrm{r} / \mathrm{min}$. The measured currents from the motor and levitation coils are shown with the measured position during the rotation test for both speeds in Fig. 13 and Fig. 14. Note, that, for illustrative purposes the rotational test are carried out only with the LQR based control approach. These results clearly indicate that the proposed control approach produces stable levitation also during rotation. Torque for the rotation is produced with the D-end motor windings without the decoupling in the levitation controller. The average fluctuation of the positon measurement during the rotation is $2.5 \mu \mathrm{m}$, which is caused by the sensor noise and the runout of the sensor surface together with the unbalance of the rotor. By comparing rotor position measurements in Fig. 13 and Fig. 14 it can be noticed that D-end orbit is affected the most from the rotation speed change. Fundamental orbit change of the rotor position with rotor speed from 150 to $300 \mathrm{r} / \mathrm{min}$ is for D-end from $2.15 \mu \mathrm{m}$ to
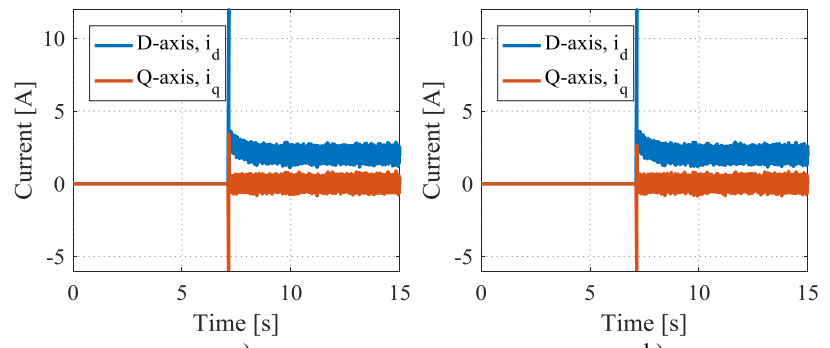

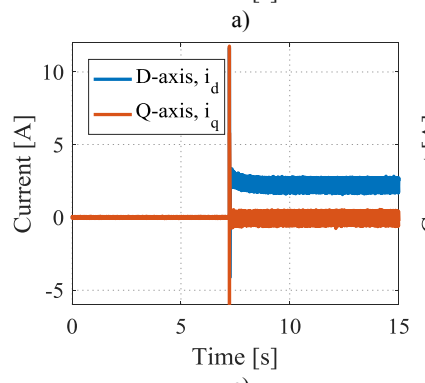

c)

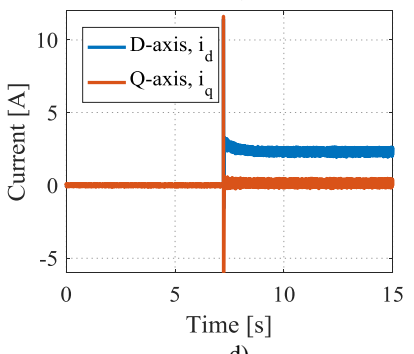

d)
Figure 11: Simulated and measured DQ currents during the rotor lift-up when using the LQR controller. Simulation of the DQ axis current of the D-end and the ND-end motor are shown in a) and b), respectively. The measured DQ axis currents of the D-end and the ND-end motor are shown in c) and d), respectively. Steady state RMS values for current in the simulation a) and b) are $2 \mathrm{~A}$. For the measured steady state RMS current values c) $2.2 \mathrm{~A}$ and d) $2.3 \mathrm{~A}$.

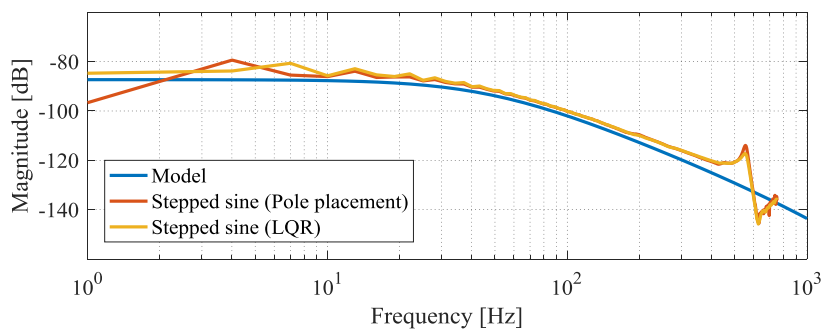

Figure 12: Frequency response plot where the result of the experimental identification with the stepped sine method is compared with the rigid body rotor model. The experimental result shows the first flexible mode peak.

$2.1 \mu \mathrm{m}$ and for ND-end $4.5 \mu \mathrm{m}$ to $3.2 \mu \mathrm{m}$. Effect of the cross-coupling between the levitation and the torque windings in D-end is seen from the results. 


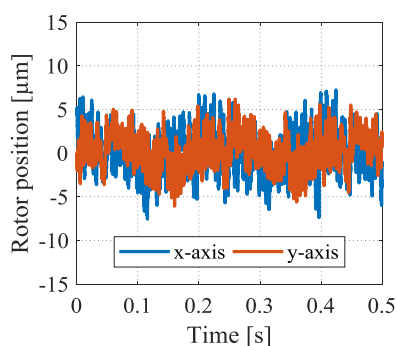

a)

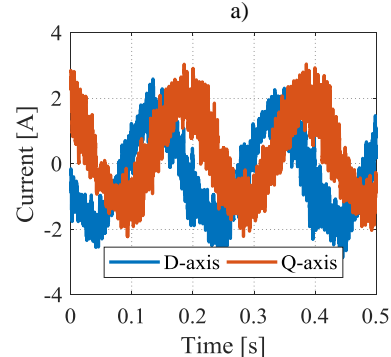

c)

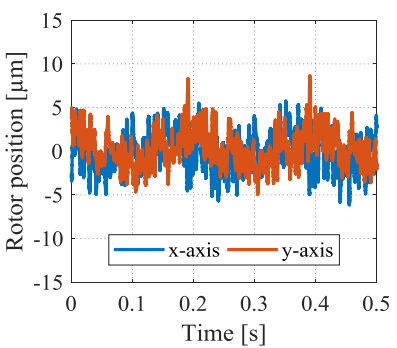

b)

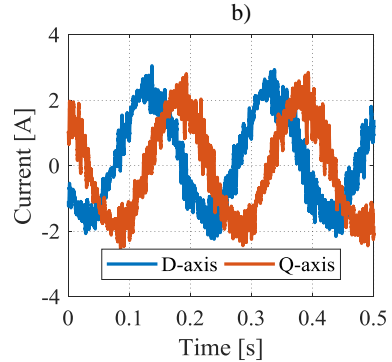

d)

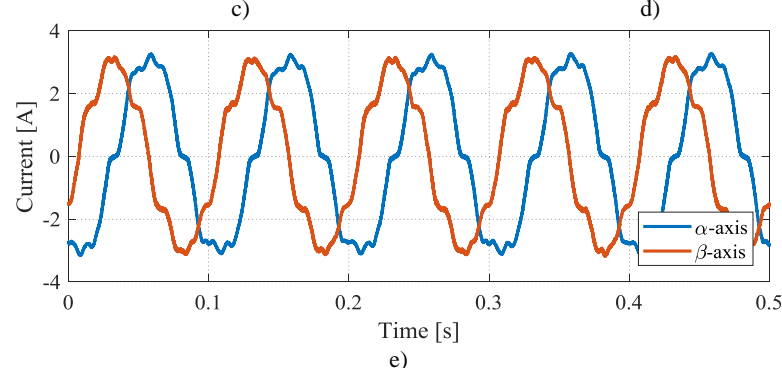

e)

Figure 13: Rotation test with speed of $150 \mathrm{r} / \mathrm{min}$. Measured rotor position is shown for D-end and ND-end in a) and b), respectively. Levitation winding current for D-end and ND-end is shown in c) and d). respectively. Torque producing current in the D-end motor windings is shown in e). The motor currents are represented in $\alpha \beta$-armature reference frame.

\section{Summary of the Commissioning Steps}

A summary of the commissioning steps is given to explicate the connection between the proposed control methods and the control system.

- Step I: Derivation of the rigid system model (2) using the rotor mass $m$ and the inertia $J$ with the position stiffness $K_{x}$ and current stiffness $K_{i}$ parameters obtained from the FEM and validated by experiments (see Fig. 5). To derive the full model used for the control design (10), the inner current controller dynamics (14) is considered.

- Step II: MIMO state space controller design considering pole placement or LQR. The initial se-
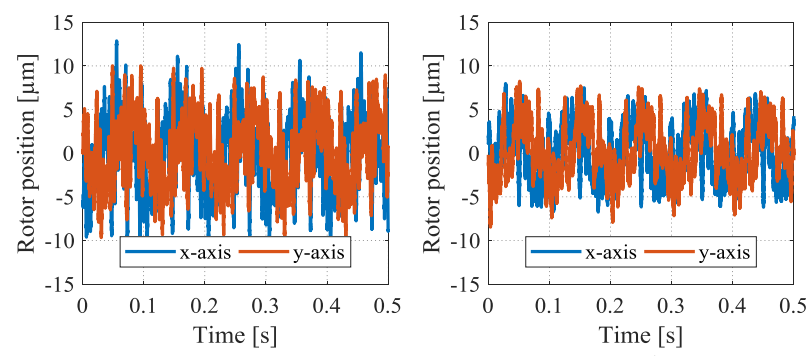

a)

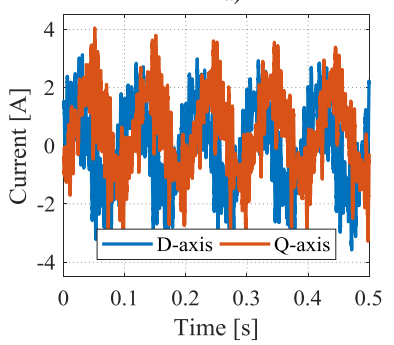

c)

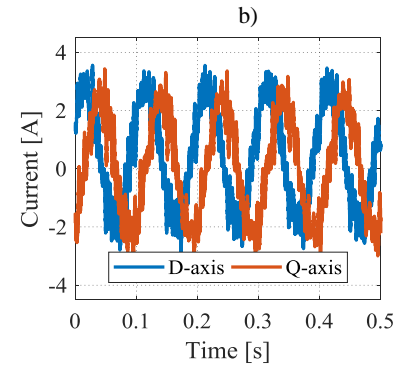

d)

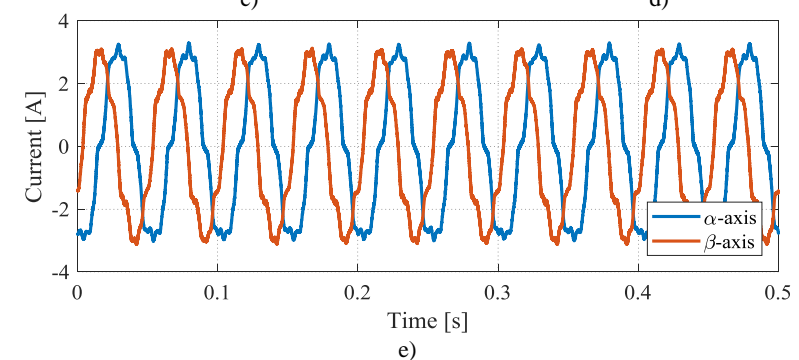

Figure 14: Rotation test with speed of $300 \mathrm{r} / \mathrm{min}$. Measured rotor position is shown for D-end and ND-end in a) and b), respectively. Levitation winding current for D-end and ND-end is shown in c) and d). respectively. Torque producing current in the $\mathrm{D}$-end motor windings is shown in e). The motor currents are represented in $\alpha \beta$-armature reference frame.

lection for the pole placement control is to place all the poles in the same location, that is, $z=$ $e^{-\sqrt{\frac{K_{x}}{m}} \cdot T_{s}}$, which corresponds to the eigenvalue for a spring-mass-system with a negative stiffness. The LQR can be straightforwardly designed with Bryson's rule by selecting reasonable maximum input signal and output deviation limits for the controller. A good initial selection for the maximum output deviation is to consider smaller values for the deviation than the values given in the ISO standard ISO 14839-2:2004(E) (2004), where the acceptable rotor vibration with respect to the air-gap length in magnetic levitation applications is recommended. Here, a value of $0.083 \cdot C_{\min }$ is considered, where $C_{\min }$ is the minimum clearance. The maximum levitation current can be used as 
the initial value for the input deviation. An acceptable control effort can be achieved by tuning the input deviation, and thus, in this paper, the selected input deviation is $2 \mathrm{~A}$.

- Step III: Estimator design (14) based on the system model, The estimator can be tuned by using the general guidelines given for instance in Messager and Binder (2016), Franklin et al. (2010) so that the observer poles are around 4-10 times faster than the closed-loop poles. Here, a ten times faster design is considered.

- Step IV: Check in the simulation that the desired response and dynamics are obtained for the lift-up test (see example in Fig. 9 a) and c)). If the requirements are not met, redesign the controller and the estimator in Steps II and III.

- Steps V-VI: Experimental lift-up test, where the basic functionality of the controller is further verified. After that, identification tests supported with a model validation routine should be carried out. Here, the adaptive amplitude stepped sine Vuojolainen et al. (2017) is used as an excitation signal in the identification experiments.

After the proposed commissioning routine, the natural next step is the controller retuning based on the identified model, if the initial mathematical model does not correspond to the identified one. This step is important, especially if there is some identified dynamics, such as cross-coupling, which should be considered in the final controller design for the rotation over the whole speed range. To this end, previous studies focusing on the control of different bearingless machine applications Zhang et al. (2016), Zhao and Zhu (2017) have shown that PID-based controllers are useful tools for stabilizing a rigid rotor. However, a MIMO controller should be considered as a final controller as it is more straightforward to tune in order to adequately stabilize the complex dynamics in the case of a flexible rotor Yoon et al. (2013). Moreover, in general, when considering a magnetically levitated high-speed motor application with a very high speed requirement, the PID controller has certain shortcomings that can destabilize the system for example if there are flexible modes within the controller bandwidth. When commissioning is carried out with a MIMO controller, the final control law can be designed using the same algorithm straightforwardly. In this case, this ensures a better cooperation between bearingless motors for the stabilization of the system and stable rotational operation over the whole speed range.

\section{Conclusion}

Commissioning steps for fully levitated bearingless machine using the model based control approach is presented. It is beneficial to apply the MIMO control principles over very traditional PID-based control structures, which do not take into account the coupling of the rotor system. In this paper, it was shown that the well-established MIMO AMB control principles can be straightforwardly applied to a bearingless machine system. By comparing the adopted controllers, it is shown that the LQR outperforms the pole placement controller. Designing an LQR-based controller is much more straightforward as a result of the more intuitive tuning methods. Secondly, weighting-function-based controllers are not sensitive to a model order change as the weights affect the inputs and outputs but not the states themselves. Updating a rigid body rotor model to a flexible model would increase the number of poles to be tuned. Naturally, the pole placement controller is more suitable for simpler systems than a complex MIMO system, such as a 4-DOF levitated rotor system, but in this paper, it was only considered as an example MIMO control case for a bearingless machine.

The results presented in this paper are important as the 5-DOF operation of bearingless machines has not been comprehensively analyzed in the literature thus far. The 5-DOF operation was shown and analyzed with two distinct MIMO control approaches using simple rigid rotor model. The proposed controllers can be applied for commissioning purposes, and it was experimentally shown that artificial-excitation-based system identification experiments can be carried out during full levitation operation. Additionally, stability of the LQR based levitation controller was verified with the low-speed rotation tests.

\section{References}

Cao, X., Yang, H., Zhang, L., and Deng, Z. Compensation strategy of levitation forces for single-winding bearingless switched reluctance motor with one winding total short circuited. IEEE Trans. on Ind. Electron., 2016. 63(9):5534-5546. doi:10.1109/TIE.2016.2558482.

Cao, X., Zhou, J., Liu, C., and Deng, Z. Advanced control method for single-winding bearingless switched reluctance motor to reduce torque ripple and radial displacement. IEEE Trans. Energy Convers., 2017. PP(99):1-1. doi:10.1109/TEC.2017.2719160.

Chen, L. and Hofmann, W. Modelling and control of one bearingless 8-6 switched reluctance motor with single layer of winding structure. In 14th European 
Conf. on Power Electron. and Appl. (EPE). pages 1-9, 2011.

Chiba, A., Fukao, T., Ichikawa, O., Oshima, M., Takemoto, M., and Dorrell, D. G. Magnetic bearings and bearingless drives, pages 1-15. Elsevier, Amsterdam, The Netherlands, 2009. doi:10.1016/B978-0-75065727-3.X5000-7.

Chiba, A., Horima, S., and Sugimoto, H. A principle and test results of a novel bearingless motor with motor parallel winding structure. In IEEE Energy Convers. Congr. and Expo. (ECCE). pages 2474-2479, 2013. doi:10.1109/ECCE.2013.6647019.

Franklin, G. F., Powell, J., and Workman, M. L. Digital control of dynamic systems, pages 364, 400401. Ellis-Kagle Press, 1200 Pilarcitos Ave. Halfmoon Bay, CA 94019, 2010.

Gerhard Schweitzer, E. H. M. Magnetic bearings, pages 1-82. Springer-Verlag Berlin Heidelberg, SpringerVerlag Berlin Heidelberg, 2009. doi:10.1007/978-3642-00497-1.

Hoshi, H., Shinshi, T., and Takatani, S. Thirdgeneration blood pumps with mechanical noncontact magnetic bearings. Artificial Organs, $2006 . \quad 30(5): 324-338$. doi:10.1111/j.15251594.2006.00222.x. Cited By 153.

Huang, J., Li, B., Jiang, H., and Kang, M. Analysis and control of multiphase permanent-magnet bearingless motor with a single set of half-coiled winding. IEEE Trans. on Ind. Electron., 2014. 61(7):31373145. doi:10.1109/TIE.2013.2279371.

ISO 14839-2:2004(E). Mechanical vibration - Vibration of rotating machinery equipped with active magnetic bearings - Part 2: Evaluation of vibration. Standard, International Organization for Standardization, Geneva, CH, 2004.

Jaatinen, P., T. Sillanpää, R. J., and Pyrhönen, O. Automated parameter identification platform for magnetic levitation systems: case bearingless machine. In 15th Int. Symp. on Magnetic Bearings. 2016.

Kauss, W. L., Gomes, A. C. D. N., Stephan, R. M., and David, D. F. B. Lqr control of a bearingless machine implemented with a dsp. In 11th Int. Symp. on Magnetic Bearings. pages 475-480, 2008.

Kolondzovski, Z., Sallinen, P., Belahcen, A., and Arkkio, A. Rotordynamic analysis of different rotor structures for high-speed permanent-magnet electrical machines. IET Electric Power Appl., 2010. 4(7):516-524. doi:10.1049/iet-epa.2008.0272.
Messager, G. and Binder, A. Observer-based pole placement control for a double conical highspeed bearingless permanent magnet synchronous motor. In 18th European Conf. on Power Electron. and Appl. (EPE). pages 1-10, 2016. doi:10.1109/EPE.2016.7695264.

Mitterhofer, H. and Amrhein, W. Motion control strategy and operational behaviour of a high speed bearingless disc drive. In 6th IET Int. Conf. on Power Electron., Mach. and Drives (PEMD). pages 1-6, 2012. doi:10.1049/cp.2012.0297.

Noh, M., Gruber, W., and Trumper, D. L. Hysteresis bearingless slice motors with homopolar flux-biasing. IEEE/ASME Transactions on Mechatronics, 2017. $\operatorname{PP}(99): 1-1$. doi:10.1109/TMECH.2017.2740429.

Ooshima, M., Chiba, A., Rahman, A., and Fukao, T. An improved control method of buriedtype ipm bearingless motors considering magnetic saturation and magnetic pull variation. IEEE Trans. Energy Convers., 2004. 19(3):569-575. doi:10.1109/TEC.2004.832065.

Ooshima, M., Kobayashi, A., and Narita, T. Stabilized suspension control strategy at failure of a motor section in a d-q axis current control bearingless motor. In IEEE Ind. Ind. Soc. Annu. Meeting. pages 1-7, 2015. doi:10.1109/IAS.2015.7356813.

Qiu, Z., Dai, J., Yang, J., Zhou, X., and Zhang, Y. Research on rotor eccentricity compensation control for bearingless surface-mounted permanentmagnet motors based on an exact analytical method. IEEE Trans. Magn., 2015. 51(11):1-4. doi:10.1109/TMAG.2015.2451163.

Schneider, T. and Binder, A. Design and evaluation of a $60000 \mathrm{rpm}$ permanent magnet bearingless high speed motor. In 7th Int. Conf. on Power Electron. and Drive Sys. (PEDS). pages 1-8, 2007. doi:10.1109/PEDS.2007.4487669.

Severson, E., Nilssen, R., Undeland, T., and Mohan, N. Design of dual purpose no voltage combined windings for bearingless motors. IEEE Trans. Ind. Appl., 2017. PP(99):1-1. doi:10.1109/TIA.2017.2706653.

Smirnov, A. AMB system for high-speed motors using automatic commissioning. Ph.D. thesis, Lappeenranta University of Technology, Lappeenranta, Finland, 2012.

Sun, X., Shi, Z., Chen, L., and Yang, Z. Internal model control for a bearingless permanent magnet synchronous motor based on inverse system method. IEEE Trans. Energy Convers., 2016a. 31(4):15391548. doi:10.1109/TEC.2016.2591925. 
Sun, X., Xue, Z., Zhu, J., Guo, Y., Yang, Z., Chen, L., and Chen, J. Suspension force modeling for a bearingless permanent magnet synchronous motor using maxwell stress tensor method. IEEE Trans. Appl. Supercond., 2016b. 26(7):1-5. doi:10.1109/TASC.2016.2599708.

Swanson, E. E., Maslen, E. H., Li, G., and Cloud, C. H. Rotordynamic design audits of amb supported machinery. In 37th Turbomachinery Symp. pages 133$158,2008$.

Takemoto, M., Iwasaki, S., Miyazaki, H., Chiba, A., and Fukao, T. Experimental evaluation of magnetic suspension characteristics in a 5-axis active control type bearingless motor without a thrust disk for wide-gap condition. In IEEE Energy Convers. Congr. and Expo. (ECCE). pages 2362-2367, 2009. doi:10.1109/ECCE.2009.5316174.

Vuojolainen, J., Nevaranta, N., Jastrzebski, R., and Pyrhönen, O. Comparison of excitation signals in active magnetic bearing system identification. Modeling, Identification and Control, 2017. 38(3):123133. doi:10.4173/mic.2017.3.2.

Warberger, B., Reichert, T., Nussbaumer, T., and Kolar, J. W. Design considerations of a bearingless motor for high-purity mixing applications. In SPEEDAM. pages 1454-1459, 2010. doi:10.1109/SPEEDAM.2010.5545102.

Xue, B., Wang, H., Tang, S., and Liang, J. Levitation performance analysis for bearingless switched reluctance motor. In 18th Int. Conf. on Elect. Machines and Syst. (ICEMS). pages 264-270, 2015. doi:10.1109/ICEMS.2015.7385039.
Yamamoto, N., Takemoto, M., Ogasawara, S., and Hiragushi, M. Experimental estimation of a 5axis active control type bearingless canned motor pump. In IEEE Int. Electric Machines and Drives Conf. (IEMDC). pages 148-153, 2011. doi:10.1109/IEMDC.2011.5994829.

Yang, S. M. and Chen, C. C. Improvements of radial force control for a spm type pmsm selfbearing motor drive (ecce). In IEEE Energy Convers. Congr. and Expo. pages 3451-3455, 2009. doi:10.1109/ECCE.2009.5316514.

Yang, Y., Deng, Z., Yang, G., Cao, X., and Zhang, Q. A control strategy for bearingless switched-reluctance motors. IEEE Trans. Power Electron., $2010 . \quad 25(11): 2807-2819$. doi:10.1109/TPEL.2010.2051684.

Yoon, Y., Lin, Z., and Allaire, P. E. Control of surge in centrifugal compressors by active magnetic bearings, pages 92-94. Springer-Verlag London, Berlin, Heidelberg, 2013. doi:10.1007/978-1-4471-4240-9.

Zhang, S., Liu, L., Wang, S., Jia, Y., and Qie, C. Complete control of radial suspension force for bearingless induction motors. In IEEE 11th Conf. on Ind. Electron. and Appl. (ICIEA). pages 2180-2184, 2016. doi:10.1109/ICIEA.2016.7603950.

Zhao, C. and Zhu, H. Design and analysis of a novel bearingless flux-switching permanent magnet motor. IEEE Trans. on Ind. Electron., 2017. 64(8):61276136. doi:10.1109/TIE.2017.2682018. 